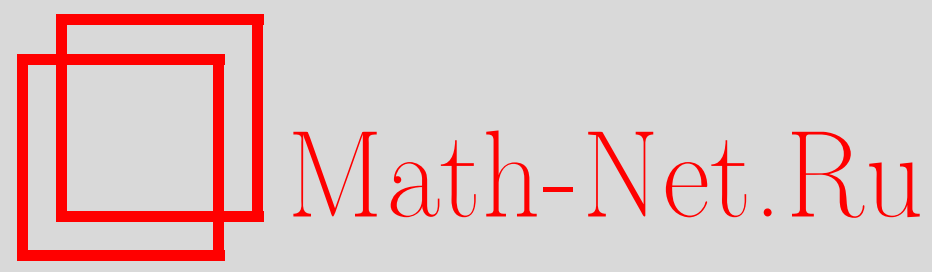

И. Т. Мамедов, Граничные свойства решений параболических уравнений второго порядка в областях со специальной симметрией, Матем. заметки, 2001, том 70, выпуск 3, 386402

DOI: https://doi.org/10.4213/mzm751

Использование Общероссийского математического портала Math-Net.Ru подразумевает, что вы прочитали и согласны с пользовательским соглашением http://www.mathnet.ru/rus/agreement

Параметры загрузки:

IP: 54.198 .67 .100

26 апреля 2023 г., 10:31:41

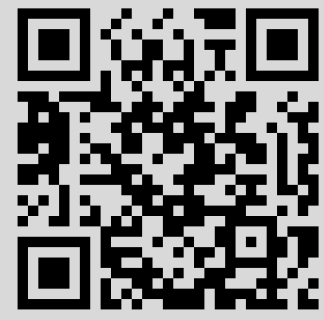


том 70 выПУСК 3 сенТяБРь 2001

УДК 517.956 .4

\section{ГРАНИЧНЫЕ СВОЙСТВА РЕШЕНИЙ \\ ПАРАБОЛИЧЕСКИХ УРАВНЕНИЙ ВТОРОГО ПОРЯДКА \\ В ОБЛАСТЯХ СО СПЕЦИАЛЬНОЙ СИММЕТРИЕЙ}

И. Т. Мамедов

Рассматривается первая краевая задача для недивергентных параболических уравнений 2-го порядка с, вообще говоря, разрьвными коэффициентами. Исследуется регулярность граничной точки в предположении, что в некоторой окрестности этой точки граница области является поверхностью вращения. В терминах параболических емкостей доказьвается необходимое и достаточное условие регулярности, смыкающееся в случае уравнения теплопроводности в критерий типа Винера.

Библиографоия: 15 названий.

Введение. Рассмотрим в ограниченной области $D$, расположенной в $(n+1)$-мерном евклидовом пространстве $\mathbb{R}_{n+1}$ точек $(x, t)=\left(x_{1}, \ldots, x_{n}, t\right)$, первую краевую задачу

$$
L u=\sum_{i, j=1}^{n} a_{i j}(x, t) u_{x_{i} x_{j}}-u_{t}=0, \quad(x, t) \in D,\left.\quad u\right|_{\Gamma(D)}=f, \quad f \in C(\Gamma(D))
$$

в предположении, что матрица $\left\|a_{i j}(x, t)\right\|$ симметрична и

$$
\begin{gathered}
\inf _{(x, t) \in D,|\xi|=1} \sum_{i, j=1}^{n} a_{i j}(x, t) \xi_{i} \xi_{j}=\lambda_{1}>0, \\
\sup _{(x, t) \in D} \sum_{i=1}^{n} a_{i i}(x, t)=M_{1}<\infty .
\end{gathered}
$$

Здесь $\Gamma(D)$ - параболическая граница области $D$. Точка $\left(x^{0}, t^{0}\right) \in \Gamma(D)$ назьвается регулярной относительно первой краевой задачи, если при всякой $f \in C(\Gamma(D))$ для обобщенного по Винеру решения $u_{f}(x, t)$ задачи (1) (см. [1]) справедливо предельное равенство

$$
\lim _{\substack{(x, t) \rightarrow\left(x^{0}, t^{0}\right) \\(x, t) \in D}} u_{f}(x, t)=f\left(x^{0}, t^{0}\right)
$$


Интерес к изучению вопросов регулярности граничной точки для параболических уравнений 2-го порядка зародился сразу после получения Н. Винером известного критерия регулярности относительно задачи Дирихле для уравнения Лапласа [2]. И. Г. Петровский [3], рассматривая одномерное уравнение теплопроводности, установил несмыкающиеся друг с другом необходимое и достаточное условия регулярности для областей, ограниченных прямыми $t=t^{1}, t=t^{2}$ и кривыми $x=\varphi_{1}(t), x=\varphi_{2}(t)$. Е. М. Ландис [4], [5] получил критерий регулярности для многомерного уравнения теплопроводности в терминах расходимости ряда из тепловых потенциалов некоторых подмножеств $\mathbb{R}_{n+1} \backslash D$. Этот критерий был перенесен на широкий класс параболических уравнений 2-го порядка в [6]-[9]. В терминах тепловых емкостей в [1], [10]-[13] были получены различные достаточные условия регулярности граничной точки. Л. Эванс и Р. Гарипи в [14] установили необходимое и достаточное условие регулярности для уравнения теплопроводности в терминах расходимости ряда из тепловых емкостей, которое можно считать полным аналогом критерия Винера для уравнения Лапласа.

Целью настоящей заметки является обобщение этого результата на произвольные равномерно параболические уравнения 2-го порядка недивергентной структуры с, вообще говоря, разрывными коэффициентами для областей, обладающих некоторой специальной симметрией.

1. Необходимое условие регулярности. Будем считать, что точка $\left(x^{0}, t^{0}\right)$ совпадает с началом координат, область $D$ расположена в полупространстве $t<0$ и в некоторой окрестности точки $(0,0)$ граница $\partial D$ представляется уравнением

$$
|x|^{2}=-t \alpha(-t), \quad-b \leqslant t<0,
$$

где $\alpha(z)$ - положительная, невозрастающая функция на $(0, b]$, при этом

$$
\left|\alpha^{\prime}(z)\right| \leqslant \frac{k}{z}, \quad z \in(0, b], \quad 0 \leqslant k<2 M_{1}
$$

Кроме того, предположим, что существует конечный или бесконечный предел

$$
J=\lim _{z \rightarrow 0+}\left|\alpha^{\prime}(z)\right| z \ln \frac{1}{z}
$$

причем если $J=4 \lambda_{1}$ и

$$
\sigma(z)=|| \alpha^{\prime}(z)\left|-\frac{4 \lambda_{1}}{z \ln (1 / z)}\right|, \quad \text { то } \quad \int_{0}^{b} \sigma(z) d z<\infty
$$

Положим для $s>0$ и $\beta>0$

$$
G_{s, \beta}(x, t)= \begin{cases}t^{-s} \exp \left(-\frac{|x|^{2}}{4 \beta t}\right) & \text { при } t>0, \\ 0 & \text { при } t \leqslant 0 .\end{cases}
$$


ЛЕмма 1 [1]. Если относительно коэффициентов оператора L выполнены условия $(2),(3), s^{+}=M_{1} /\left(2 \lambda_{1}\right), \beta^{+}=\lambda_{1} u(y, \tau)-$ произвольная точка $D$, то для $(x, t) \in D \backslash\{(y, \tau)\}$

$$
L_{(x, t)} G_{s^{+}, \beta^{+}}(x-y, t-\tau) \geqslant 0 .
$$

Для доказательства заметим, что для $t>\tau$

$$
\begin{aligned}
L G_{s^{+}, \beta^{+}}= & \frac{G_{s^{+}, \beta^{+}}}{t-\tau}\left(\frac{1}{4\left(\beta^{+}\right)^{2}(t-\tau)} \sum_{i, j=1}^{n} a_{i j}(x, t)\left(x_{i}-y_{i}\right)\left(x_{j}-y_{j}\right)\right. \\
& \left.-\frac{1}{2 \beta^{+}} \sum_{i=1}^{n} a_{i i}(x, t)+s^{+}-\frac{|x-y|^{2}}{4 \beta^{+}(t-\tau)}\right) \\
\geqslant & \frac{G_{s^{+}, \beta^{+}}}{t-\tau}\left(\frac{\lambda_{1}|x-y|^{2}}{4\left(\beta^{+}\right)^{2}(t-\tau)}-\frac{M_{1}}{2 \beta^{+}}+s^{+}-\frac{|x-y|^{2}}{4 \beta^{+}(t-\tau)}\right)=0 .
\end{aligned}
$$

СЛЕДСТВИЕ 1. Из условий (2), (3) следует, что

$$
\sup _{(x, t) \in D,|\xi|=1} \sum_{i, j=1}^{n} a_{i j}(x, t) \xi_{i} \xi_{j}=\lambda_{2}<\infty, \quad \inf _{(x, t) \in D} \sum_{i=1}^{n} a_{i i}(x, t)=M_{2}>0 .
$$

Тогда если $s^{+}=M_{2} /\left(2 \lambda_{2}\right), \beta^{-}=\lambda_{1}$ и $(y, \tau)$-произвольная точка $D$, то для $(x, t) \in$ $D \backslash\{(y, \tau)\}$

$$
L_{(x, t)} G_{s^{-}, \beta^{-}}(x-y, t-\tau) \leqslant 0 .
$$

В дальнейшем $G_{s^{ \pm}, \beta^{ \pm}}(x, t)$ будем обозначать через $G_{ \pm}(x, t)$. Запись $C(\cdots)$ означает, что положительная константа $C$ зависит лишь от содержимого скобок.

ОПрЕДЕЛЕниЕ 1 . Пусть $K-$ компакт в $\mathbb{R}_{n+1}$. Назовем меру $\mu^{ \pm}$с носителем в $K$ $\left(s^{ \pm}, \beta^{ \pm}\right)$-допустимой, если

$$
\int_{K} G^{ \pm}(x-y, t-\tau) d \mu^{ \pm}(y, \tau) \leqslant 1 \quad \text { при }(x, t) \notin K .
$$

Число $p^{ \pm}(K)=\sup \mu^{ \pm}(K)$, где точная верхняя грань берется по всем $\left(s^{ \pm}, \beta^{ \pm}\right)$-допустимым мерам, называется $G^{ \pm}$-емкостью компакта $K$. В случае $s^{+}=s^{-}=n / 2$, $\beta^{+}=\beta^{-}=1$ соответствуюшая емкость называется тепловой и обозначается чеpeз $p(K)$.

Пусть $t^{0}<t^{1}<t^{2}<\cdots<t^{k}, \Omega_{i}$ - ограниченные $n$-мерные области, $F_{i}$ - цилиндры $\Omega_{i} \times\left(t^{i-1}, t^{i}\right), i=1, \ldots, k$. Область $Q$ назьвается ступенчатой, если она представляет собой множество внутренних точек объединения $\bigcup_{i=1}^{k} \bar{F}_{i}$. При этом

$$
S(Q)=\bigcup_{i=1}^{k}\left(\partial \Omega_{i} \times\left[t^{i-1}, t^{i}\right]\right) .
$$

Для произвольной $(n+1)$-мерной области $H$ через $\gamma(H)$ обозначим совокупность всех точек $(x, t) \in \partial H$, для каждой из которых существует $r=r(x, t)$ такое, что $C_{x, r}^{t-r, t} \subset H$, $C_{x, r}^{t, t+r} \subset \mathbb{R}_{n+1} \backslash H$. Здесь $C_{x^{0}, r}^{a, b}$ - цилиндр, определяемьй неравенствами $\left|x-x^{0}\right|<r$, $a<t<b$.

Множество $\Gamma(H)=\partial H \backslash \gamma(H)$ называется параболической граничей области $H$.

Пусть $V$ - область в $\mathbb{R}_{n+1},\left(x^{0}, t^{0}\right) \in V$. Обозначим через $\Lambda\left[\left(x^{0}, t^{0}\right)\right]$ совокупность точек $(x, t) \in V \backslash\left\{\left(x^{0}, t^{0}\right)\right\}$, для каждой из которых существует непрерьвная кривая, соединяющая точки $(x, t)$ и $\left(x^{0}, t^{0}\right)$, такая, что вдоль этой кривой при движении от $(x, t)$ к $\left(x^{0}, t^{0}\right)$ координата $t$ строго возрастает. 
ОпреДЕЛЕниЕ 2. Определенная в области $V$ полунепрерывная снизу функция $u(x, t)$ назьвается $L$-суперпараболической, если

а) $-\infty<u(x, t) \leqslant+\infty$;

в) для каждой точки $(x, t) \in V$ существует точка $\left(x^{0}, t^{0}\right) \in V$ такая, что $(x, t) \in$ $\Lambda\left[\left(x^{0}, t^{0}\right)\right]$ и $u\left(x^{0}, t^{0}\right)<+\infty$;

c) для всякой строго внутренней подобласти $V^{\prime}$ области $V$ и произвольного решения $h(x, t)$ уравнения $L h=0$ в $V^{\prime}$ из $u-\left.h\right|_{\Gamma\left(V^{\prime}\right)} \geqslant 0$ следует $u \geqslant h$ в $V^{\prime}$.

Функция $u(x, t)$ назьвается $L$-субпараболической в $V$, если функция $-u(x, t)$ является $L$-суперпараболической в $V$.

Пусть для натурального $m$

$$
A_{m}^{ \pm}=\left\{(x, t): e^{m s^{ \pm}} \leqslant G^{ \pm}(-x,-t) \leqslant e^{(m+1) s^{ \pm}}\right\}, \quad B_{m}^{ \pm}=A_{m}^{ \pm} \backslash D .
$$

Всюду в дальнейшем мы будем предполагать, что обобщенное по Винеру решение $u_{f}(x, t)$ задачи (1) существует. Для этого достаточно, например, чтобы в каждой строго внутренней подобласти области $D$ коэффициенты оператора $L$ удовлетворяли равномерному условию Дини.

Теорема 1. Если коэффициенты оператора $L$ удовлетворяют в области $D$ условиям (2), (3), то для регулярности точки $(0,0)$ относительно первой краевой задачи необходимо, чтобь

$$
\sum_{m=1}^{\infty} e^{m s^{-}} p^{-}\left(B_{m}^{-}\right)=\infty
$$

ДокАЗАТЕЛЬСтво. Пусть условие (6) не вьполнено. Обозначим через $m_{1}$ наименьшее натуральное число, для которого

$$
\sum_{m=m_{1}}^{\infty} e^{m s^{-}} p^{-}\left(B_{m}^{-}\right) \leqslant \frac{e^{-s^{-}}}{4} .
$$

Выберем непрерывную граничную функцию $f(x, t)$ первой краевой задачи (1) так, чтобы $f(0,0)=1, f(x, t)=0$ при $t \leqslant \inf _{(y, \tau) \in B_{m_{1}}^{-}}\{\tau\}, 0 \leqslant f(x, t) \leqslant 1$. Согласно [15] для каждого натурального $m \geqslant m_{1}$ при любом $\varepsilon_{m}>0$ существуют ступенчатая область $Q_{m} \supset B_{m}^{-}$с достаточно гладкими границами оснований составляюших цилиндров и мера $\mu_{m}$ с носителем в $\overline{Q_{m}}$ такие, что если

$$
U_{m}(x, t)=\int_{\overline{Q_{m}}} G^{-}(x-y, t-\tau) d \mu_{m}(y, \tau),
$$

то

$$
\begin{gathered}
\left.U_{m}\right|_{S\left(Q_{m}\right) \cup \gamma\left(Q_{m}\right)}=1, \\
\mu_{m}\left(\overline{Q_{m}}\right) \leqslant p^{-}\left(B_{m}^{-}\right)+\varepsilon_{m} .
\end{gathered}
$$


Положим $\varepsilon_{m}=e^{-s^{-}} 2^{-m} / 4$. Согласно следствию из леммы 1 и принципу максимума функция $U(x, t)=\sum_{m=m_{1}}^{\infty} U_{m}(x, t)$ является $L$-суперпараболической в

$$
D^{1}=D \backslash \overline{\bigcup_{m=m_{1}}^{\infty} Q_{m}}
$$

Кроме того, из (8) с учетом выбора функции $f(x, t)$ следует, что

$$
u_{f}(x, t) \leqslant U(x, t) \quad \text { для }(x, t) \in \Gamma\left(D^{1}\right) .
$$

По принщипу максимума $u_{f}(x, t) \leqslant U(x, t)$ в $D^{1}$. Поэтому, учитьвая $(7)$ и $(9)$, получаем

$$
\begin{aligned}
\varlimsup_{\substack{(x, t) \rightarrow(0,0) \\
(x, t) \in D^{1}}} u_{f}(x, t) & \leqslant U(0,0) \leqslant \sum_{m=m_{1}}^{\infty} e^{(m+1) s^{-}} \mu_{m}\left(\overline{Q_{m}}\right) \\
& \leqslant e^{s^{-}}\left(\sum_{m=m_{1}}^{\infty} e^{m s^{-}} p^{-}\left(B_{m}^{-}\right)+\frac{e^{-s^{-}}}{4} \sum_{m=m_{1}}^{\infty} 2^{-m}\right) \leqslant \frac{1}{2},
\end{aligned}
$$

т.е. точка $(0,0)$ является иррегулярной. Теорема доказана.

2. Некоторые емкостные оценки. Для натурального $m$ обозначим через $z_{m}$ корень уравнения

$$
\alpha\left(z_{m}\right)=2 M_{1} \ln \frac{e^{-m}}{z_{m}} .
$$

Покажем вначале, что для достаточно больших $m$ уравнение (10) имеет корень в интервале $\left(e^{-\gamma_{1} m}, e^{-m}\right)$, где $\gamma_{1}=4 M_{1} /\left(2 M_{1}-K\right)$. Действительно, если

$$
\varphi(z)=\alpha(z)-2 M_{1} \ln \frac{e^{-m}}{z}
$$

то

$$
\begin{gathered}
\varphi\left(e^{-m}\right)=\alpha\left(e^{-m}\right)>0, \\
\varphi\left(e^{-\gamma_{1} m}\right)=\alpha\left(e^{-m}\right)-2 M_{1}\left(\gamma_{1}-1\right)^{m} .
\end{gathered}
$$

С другой стороны, для $t^{1} \geqslant t^{2}>0$ из (4) следует, что

$$
\alpha\left(t^{2}\right)-\alpha\left(t^{1}\right) \leqslant K \ln \frac{t^{1}}{t^{2}}
$$

т.е. для достаточно малых $t$

$$
\alpha(t) \leqslant \alpha(b)+K \ln b+K \ln \frac{1}{t}<\frac{2 M_{1}+K}{2} \ln \frac{1}{t} .
$$

Теперь из (12) получаем

$$
\varphi\left(e^{-\gamma_{1} m}\right)<m\left(\frac{2 M_{1}+K}{2} \gamma_{1}-2 M_{1}\left(\gamma_{1}-1\right)\right)=0,
$$


что вместе с (11) гарантирует наличие корня в указанном интервале. Если уравнение (10) имеет более одного корня в интервале $\left(e^{-\gamma_{1} m}, e^{-m}\right)$, то через $z_{m}$ обозначим точную нижнюю грань всех этих корней. Заметим, что $z_{m}$ является модулем временной координаты точек пересечения поверхности уровня $G^{+}(-x,-t)=e^{m s^{+}}$с $\partial D$.

Нетрудно видеть, что для достаточно больших $m$ последовательность $\left\{z_{m}\right\}$ не возрастает. Действительно, из (10) следует, что

$$
\frac{z_{m}}{z_{m+1}}=\exp \left(-\frac{1}{2 M-1}\left(\alpha\left(z_{m}\right)-\alpha\left(z_{m+1}\right)\right)+1\right)
$$

Если теперь предположить, что $z_{m}<z_{m+1}$, то из (13) и (14) вьводим

$$
\frac{z_{m}}{z_{m+1}} \geqslant e \cdot\left(\frac{z_{m}}{z_{m+1}}\right)^{K /\left(2 M_{1}\right)}
$$

т.e.

$$
\frac{z_{m}}{z_{m+1}} \geqslant \exp \left(\frac{2 M_{1}}{2 M_{1}-K}\right)>1,
$$

и мы получаем противоречие. Итак $z_{m} \geqslant z_{m+1}$, что с учетом (13) и (14) влечет за собой

$$
e^{-d} z_{m} \leqslant z_{m+1} \leqslant e^{-1} z_{m}
$$

где $d=2 M_{1} /\left(2 M_{1}-K\right)$. Обозначим $e^{-m} z_{m}$ через $r_{m}$. Из (15) заключаем, что для достаточно больших $m r_{m} \geqslant r_{m+1}$, т.е. существует предел $r_{0}=\lim _{m \rightarrow \infty} r_{m}$. Не теряя в общности, будем считать, что $r_{0}<1$.

ЛЕмма 2. Если относительно области D выполнено условие (4), то для достаточно больиих $\mathrm{m}$

$$
p^{+}\left(B_{m}^{+}\right) \leqslant C_{1}\left(\alpha, s^{+}, \beta^{+}, n\right)\left(z_{m}\right)^{s^{+}}\left(\ln \frac{1}{r_{m}}\right)^{n-s^{+}} .
$$

ДокАЗАТЕЛЬСТВо. Мы ограничимся рассмотрением случая $r_{0}=0$. При $r_{0}>0$ доказательство проводится по аналогичной схеме. Для натурального $m$ обозначим через $T_{m}$ пересечение $B_{m}^{+} \cap\left\{(x, t):-z_{m} \leqslant t \leqslant-z_{m+1}\right\}$. Пусть $\tau \in\left[-z_{m}, z_{m+1}\right]$. Обозначим через $h_{m}(\tau)$ длину отрезка, параллельного оси $t$, соединяющего точку лежащей в гиперплоскости $t=\tau$ сфферы

$$
|y|=\sqrt{2 M_{1}(-\tau) \ln \frac{e^{-m}}{-\tau}}
$$

с соответствующей точкой поверхности уровня $G^{+}(-x,-t)=e^{(m+1) s^{+}}$.

Имеем

$$
\sqrt{2 M_{1}(-\tau) \ln \frac{e^{-m}}{-\tau}}=\sqrt{2 M_{1}\left(-\tau+h_{m}(\tau)\right) \ln \frac{e^{-m-1}}{-\tau+h_{m}(\tau)}}
$$


Обозначая $h_{m}(\tau) /|\tau|$ через $v_{m}(\tau)$, из предыдущего равенства получаем

$$
\begin{aligned}
v_{m}(\tau) & =\frac{1}{\ln \left(e^{-m} /(-\tau)\right)}\left(1+v_{m}(\tau)+v_{m}(\tau) \ln \left(1+v_{m}(\tau)\right)+\ln \left(1+v_{m}(\tau)\right)\right) \\
& \leqslant \frac{2+2 \ln 2+\ln 2}{\ln \left(e^{-m} / z_{m}\right)} \leqslant \frac{5}{\ln \left(1 / r_{m}\right)}
\end{aligned}
$$

откуда следует, что

$$
h_{m}(\tau) \leqslant \frac{5}{\ln \left(1 / r_{m}\right)} .
$$

С другой стороны,

$$
\sqrt{2 M_{1} z_{m} \ln \frac{e^{-m}}{z_{m}}}-\sqrt{2 M_{1} z_{m} \ln \frac{e^{-m-1}}{z_{m}}} \leqslant \sqrt{2 M_{1} \frac{z_{m}}{\ln \left(1 / r_{m}\right)}} .
$$

Поэтому если

$$
C_{2}=\max \left\{\sqrt{5}, \sqrt{2 M_{1}}\right\}, \quad \rho_{m}=C_{2} \sqrt{\frac{z_{m}}{\ln \left(1 / r_{m}\right)}},
$$

то из (17) и (18) заключаем, что $T_{m}$ можно покрыть $C_{3}\left(\alpha, s^{+}, \beta^{+}, n\right) \cdot\left[\ln \left(1 / r_{m}\right)\right]^{n}$ числом цилиндров вида $C_{x^{1}, \rho_{m}}^{t^{1}-\rho_{m}^{2}, t^{1}}$. Согласно [1]

$$
p^{+}\left(C_{x^{1}, \rho_{m}}^{t^{1}-\rho_{m}^{2}, t^{1}}\right)=C_{4}\left(C_{2}, s^{+}, \beta^{+}, n\right) \cdot \rho_{m}^{2 s^{+}} .
$$

Из полуаддитивности $G^{+}$-емкости и $(19)$ получаем

$$
p^{+}\left(T_{m}\right) \leqslant C_{5}\left(\alpha, s^{+}, \beta^{+}, n\right) \cdot\left(z_{m}\right)^{s^{+}}\left(\ln \frac{1}{r_{m}}\right)^{n-s^{+}} .
$$

Совершенно аналогично можно показать, что если для фиксированного $m$ и любого целого неотрицательного $i T_{m, i}=B_{m}^{+} \cap\left\{(x, t):-z_{m+i} \leqslant t \leqslant-z_{m+i+1}\right\}$, то

$$
p^{+}\left(T_{m, i)} \leqslant C_{5}\left(z_{m+i}\right)^{s^{+}}\left(\ln \frac{1}{r_{m+i}}\right)^{n-s^{+}}\right.
$$

Поэтому

$$
p^{+}\left(B_{m}^{+}\right) \leqslant \sum_{i=0}^{\infty} p^{+}\left(T_{m, i)} \leqslant C_{5}\left(z_{m}\right)^{s^{+}}\left(\ln \frac{1}{r_{m}}\right)^{n-s^{+}} \sum_{i=0}^{\infty} a_{m, i}\right.
$$

где

$$
a_{m, i}=\left(\frac{z_{m+i}}{z_{m}}\right)^{s^{+}}\left(\frac{\ln \left(1 / r_{m+i}\right)}{\ln \left(1 / r_{m}\right)}\right)^{n-s^{+}} .
$$

Так как последовательность $\left\{1 / r_{m}\right\}$ не убывает, то при $s^{+} \geqslant n$

$$
\frac{a_{m, i+1}}{a_{m, i}} \leqslant e^{-s^{+}}
$$


поэтому

$$
\sum_{i=0}^{\infty} a_{m, i} \leqslant \frac{a_{m, 0}}{1-e^{-s^{+}}}=\frac{1}{1-e^{-s^{+}}} .
$$

Пусть $s^{+}<n$. Согласно (15) имеем

$$
\ln \frac{1}{r_{m+i+1}} \leqslant \ln \frac{1}{r_{m+i}}+d-1
$$

что при достаточно больших $m$ и предположении $r_{0}=0$ влечет за собой оценку

$$
\left(\frac{\ln \left(1 / r_{m+i+1}\right)}{\ln \left(1 / r_{m+i}\right)}\right)^{n-s^{+}} \leqslant\left(1+\varepsilon_{0}\right)^{n-s^{+}}
$$

где $\varepsilon_{0}=\exp \left(s^{+} /\left(2\left(n-s^{+}\right)\right)-1\right.$. Тогда

$$
\frac{a_{m, i+1}}{a_{m, i}} \leqslant e^{-s^{+} / 2}
$$

и, следовательно,

$$
\sum_{i=0}^{\infty} a_{m, i} \leqslant \frac{1}{1-e^{-s^{+} / 2}} .
$$

Теперь из (20)-(22) вытекает требуемая оценка (16) с $C_{1}=C_{5} /\left(1-e^{-s^{+} / 2}\right)$. Лемма доказана.

Пусть для натурального $m H_{m}=B_{m}^{+} \cap\left\{(x, t): t \leqslant-z_{m+2}\right\}$.

ЛЕмма 3. Если относительно области $D$ выполнено условие (4), то для достаточно больиих $\mathrm{m}$

$$
p^{+}\left(H_{m}\right) \geqslant C_{6}\left(\alpha, s^{+}, \beta^{+}, n\right)\left(z_{m}\right)^{s^{+}}\left(\ln \frac{1}{r_{m}}\right)^{n-s^{+}} .
$$

ДокАЗАТЕЛьство. Так же, как и в предыдущей лемме, ограничимся случаем $r_{0}=0$. Пусть для натурального $m$

$$
\theta_{m}=\sqrt{2 M_{1} z_{m+2} \ln \frac{e^{-m}}{z_{m+2}}}-\sqrt{2 M_{1} z_{m+2} \ln \frac{e^{-m-1}}{z_{m+2}}} .
$$

Нетрудно видеть, что

$$
\sqrt{\frac{M_{1}}{2} \frac{z_{m+2}}{\ln \left(e^{-m} / z_{m+2}\right)}} \leqslant \theta_{m} \leqslant \sqrt{2 M_{1} \frac{z_{m+2}}{\ln \left(e^{-m} / z_{m+2}\right)}} .
$$

Обозначим через $H_{m}^{1}$ множество

$$
\left\{(x, t): \sqrt{2 M_{1}(-t) \ln \frac{e^{-m}}{-t}}-\theta_{m} \leqslant|x| \leqslant \sqrt{2 M_{1}(-t) \ln \frac{e^{-m}}{-t}}\right\}, \quad m=1,2, \ldots
$$


Так как $H_{m}^{1} \subset H_{m}$, то

$$
p^{+}\left(H_{m}\right) \geqslant p^{+}\left(H_{m}^{1}\right) .
$$

Пусть далее для натурального $m$

$$
S_{m}=\left\{(y, \tau):|y|=\sqrt{2 M_{1}(-\tau) \ln \frac{e^{-m}}{-\tau}}-\frac{\theta}{2}, \quad-z_{m+1}+\theta_{m}^{2} \leqslant \tau \leqslant-z_{m+2}-\theta_{m}^{2}\right\} .
$$

В силу нашего предположения относительно $r_{0}$ и (24)

$$
\frac{z_{m+1}-z_{m+2}}{\theta_{m}^{2}} \rightarrow \infty \quad \text { при } \quad m \rightarrow \infty
$$

поэтому $S_{m} \subset H_{m}^{1}$. Зафиксируем точку $(x, t)$ на боковой поверхности $H_{m}^{1}$ и обозначим через $S_{m}^{1}$ ту часть $S_{m}$, из которой удалены точки $(y, \tau)$, где $0<t-\tau<\theta_{m}^{2} /\left(4 M_{1}\right)$. Если $I_{1}=\int_{S_{m}^{1}} G^{+}(x-y, t-\tau) d S_{(y, \tau)}$, то, очевидно, $I_{1}=0$ при $t \leqslant-z_{m+1}+\theta_{m}^{2}$. Пусть $t>-z_{m+1}+\theta_{m}^{2}$. Перейдем от интегрирования по поверхности $S_{m}^{1}$ к интегрированию по ее проекции $S_{m}^{2}$ на гиперплоскость $\tau=-z_{m+1}+\theta_{m}^{2}$. Обозначая через $\left(x^{\prime}, t^{\prime}\right)$ и $(v, \eta)$ образы точек $(x, t)$ и $(y, \tau)$ соответственно, получаем

$$
\begin{aligned}
I_{1} & =\left(t^{\prime}-\eta\right)^{-s^{+}} \int_{S_{m}^{2}} \exp \left(-\frac{\left|x^{\prime}-v\right|^{2}}{4 \beta^{+}\left(t^{\prime}-\eta\right)}\right) \sqrt{1+\left(\frac{\partial \eta}{\partial v_{1}}\right)^{2}+\cdots+\left(\frac{\partial \eta}{\partial v_{n}}\right)^{2}} d v \\
& =\left(t^{\prime}-\eta\right)^{-s^{+}} \int_{S_{m}^{2}} \exp \left(-\frac{\left|x^{\prime}-v\right|^{2}}{4 \beta^{+}\left(t^{\prime}-\eta\right)}\right) \sqrt{1+\left(\frac{\partial \eta}{\partial|v|}\right)^{2}} d v .
\end{aligned}
$$

Но, с другой стороны, из равенства $r_{0}=0$ следует, что

$$
\frac{\partial \eta}{\partial|v|}=\left(\frac{\partial|v|}{\partial \eta}\right)^{-1} \leqslant \sqrt{\frac{2}{M_{1}} \frac{|\eta| \ln \left(e^{-m} /(-\eta)\right)}{\left(\ln \left(e^{-m} /(-\eta)\right)-1\right)^{2}}} \leqslant \frac{2}{\sqrt{M_{1}}},
$$

если только $m$ достаточно велико. Используя эту оценку и тот факт, что $s^{+} \geqslant n / 2$, из (26) заключаем, что

$$
\begin{aligned}
I_{1} & \leqslant C_{7}\left(M_{1}, n\right)\left(t^{\prime}-\eta\right)^{-s^{+}} \int_{S_{m}^{2}} \exp \left(-\frac{\left|x^{\prime}-v\right|^{2}}{4 \beta^{+}\left(t^{\prime}-\eta\right)}\right) d v \\
& \leqslant C_{8}\left(M_{1}, n\right) \theta_{m}^{-2 s^{+}+n}\left(\int_{-\infty}^{+\infty} e^{-\xi^{2}} d \xi\right)^{n}=C_{9}\left(M_{1}, n\right) \theta_{m}^{-2 s^{+}+n} .
\end{aligned}
$$

Пусть теперь $S_{m}^{3}=S_{m} \backslash S_{m}^{1},(y, \tau) \in S_{m}^{3}$. Покажем, что если точка $(x, t)$ расположена на боковой поверхности $H_{m}^{1}$ и $0<t-\tau<\theta_{m}^{2} /\left(4 M_{1}\right)$, то для достаточно больших $m$

$$
|x-y| \geqslant \frac{\theta_{m}}{4}
$$

Действительно, пусть, например, точка $(x, t)$ расположена на поверхности

$$
|x|=\sqrt{2 M_{1}(-t) \ln \frac{e^{-m}}{-t}} .
$$


Имеем

$$
\begin{aligned}
|x-y| & \geqslant|x|-|y|=\sqrt{2 M_{1}(-t) \ln \frac{e^{-m}}{-t}}-\sqrt{2 M_{1}(-\tau) \ln \frac{e^{-m}}{-\tau}}+\frac{\theta_{m}}{2} \\
& \geqslant \frac{\theta_{m}}{2}-\sqrt{\frac{M_{1}}{2}} \frac{\left|\ln \left(e^{-m} /(-\xi)\right)-1\right|}{\sqrt{-\xi \ln \left(e^{-m} /(-\xi)\right)}}(t-\tau),
\end{aligned}
$$

где $\tau<\xi<t$. Теперь, учитывая (24) и то, что $r_{0}=0$, для достаточно больших $m$ получаем

$$
|x-y| \geqslant \frac{\theta_{m}}{2}-\sqrt{\frac{M_{1}}{2} \frac{\ln \left(e^{-m} / z_{m+2}\right)}{z_{m+2}}}(t-\tau) \geqslant \frac{\theta_{m}}{2}-\frac{M_{1}}{\theta_{m}}(t-\tau) \geqslant \frac{\theta_{m}}{4} .
$$

Аналогично доказьвается неравенство (28), если точка $(x, t)$ расположена на поверхности $|x|=\sqrt{2 M_{1}(-t) \ln \left(e^{-m} /(-t)\right)}-\theta_{m}$. Имеем

$$
\begin{aligned}
I_{2} & =\int_{S_{m}^{3}} G^{+}(x-y, t-\tau) d S_{(y, \tau)} \\
& \leqslant 4^{2 s^{+}} \theta_{m}^{-2 s^{+}} \int_{S_{m}^{3}}\left(\frac{|x-y|^{2}}{t-\tau}\right)^{s^{+}} \exp \left(-\frac{|x-y|^{2}}{4 \beta^{+}(t-\tau)}\right) d S_{(y, \tau)} .
\end{aligned}
$$

Переходя теперь к интегрированию по проекции $S_{m}^{3}$ на гиперплоскость $t=-z_{m+1}+\theta_{m}^{2}$ и учитьвая, что

$$
\int_{\mathbb{R}_{n+1} \times\left\{t=-z_{m+1}+\theta_{m}^{2}\right\}}|w|^{2 s^{+}} \exp \left(-\frac{|w|^{2}}{4 \beta^{+}}\right) d w<\infty,
$$

получаем

$$
I_{2} \leqslant C_{10}\left(s^{+}, \beta^{+}, n\right) \theta_{m}^{-2 s^{+}+n} .
$$

Используя те же соображения, что и при оценке интеграла $I_{1}$, заключаем, что

$$
I=\int_{S_{m}} G^{+}(x-y, t-\tau) d S_{(y, \tau)} \leqslant C_{9} \theta_{m}^{-2 s^{+}+n}
$$

для $(x, t) \in \gamma\left(H_{m}^{1}\right)$. Из $(27),(29)$ и $(30)$ следует, что для $(x, t) \in \partial H_{m}^{1}$

$$
I \leqslant\left(C_{9}+C_{10}\right) \theta_{m}^{-2 s^{+}+n} .
$$

Поэтому, учитьвая (24) и (15), для достаточно больших $m$ получаем

$$
\begin{aligned}
p^{+}\left(H_{m}^{1}\right) & \geqslant \frac{\theta_{m}^{2 s^{+}-n}}{C_{9}+C_{10}} \int_{S_{m}} d S_{(y, \tau)} \geqslant \frac{C_{11}\left(\alpha, M_{1}, n\right)}{C_{9}+C_{10}} \theta_{m}^{2 s^{+}-n}\left(z_{m+2} \ln \frac{e^{-m}}{z_{m+2}}\right)^{n / 2} \\
& \geqslant C_{12}\left(\alpha, s^{+}, \beta^{+}, n\right)\left(z_{m+2}\right)^{s^{+}}\left(\ln \frac{e^{-m}}{z_{m+2}}\right)^{n-s^{+}} \\
& \geqslant C_{12} e^{-2 d s^{+}} 2^{-\left|n-s^{+}\right|}\left(z_{m}\right)^{s^{+}}\left(\ln \frac{1}{r_{m}}\right)^{n-s^{+}} .
\end{aligned}
$$

Из последнего неравенства и (25) вытекает требуемая оценка (23) с $C_{6}=C_{12} e^{-2 d s^{+}} \times$ $2^{-\left|n-s^{+}\right|}$. Лемма доказана. 


\section{3. Теорема о возрастании положительных решений.}

ТЕОРема 2. Если относительно области D выполнены предположения (4) и (5), то из условия

$$
\sum_{m=1}^{\infty} e^{m s^{+}} p^{+}\left(B_{m}^{+}\right)=\infty
$$

следует, что

$$
\sum_{m=3}^{\infty} \frac{1}{\ln \ln m} e^{m s^{+}} p^{+}\left(B_{m}^{+}\right)=\infty
$$

ДоКАЗАТЕЛЬСТво. Пусть выполнено условие (31). Из лемм 2 и 3 заключаем, что (31) эквивалентно условию

$$
\sum_{m=1}^{\infty}\left(r_{m}\right)^{s^{+}}\left(\ln \frac{1}{r_{m}}\right)^{n-s^{+}}=\infty
$$

Рассмотрим случай, когда константа $J$ в (5) равна $4 \lambda_{1}$, и предположим вначале, что $s^{+} \leqslant n+1$. Считая $b<e^{-1}$, из $(5)$ для $z \in(0, b)$ находим

$$
\alpha(z) \leqslant \alpha(b)+4 \lambda_{1} \ln \ln \frac{1}{z}+\int_{0}^{b} \sigma(t) d t,
$$

откуда следует, что

$$
r_{m}=\exp \left(-\frac{\alpha\left(z_{m}\right)}{2 M_{1}}\right) \geqslant C_{13}\left(\alpha, M_{1}\right)\left(\ln \frac{1}{z_{m}}\right)^{-2 \lambda_{1} / M_{1}} .
$$

Но так как $z_{m} \geqslant e^{-\gamma_{1} m}$, то

$$
\left(r_{m}\right)^{s^{+}} \geqslant C_{14}\left(\alpha, M_{1}, s^{+}\right) m^{-\left(2 \lambda_{1} / M_{1}\right) s^{+}}=C_{14} m^{-1} .
$$

Если $s^{+} \leqslant n$, то из $(33)$ вытекает расходимость ряда

$$
\sum_{m=3}^{\infty} \frac{1}{\ln \ln m}\left(r_{m}\right)^{s^{+}}\left(\ln \frac{1}{r_{m}}\right)^{n-s^{+}},
$$

т.е. в этом случае теорема доказана. Пусть $s^{+} \in(n, n+1]$. Из (33) следует, что

$$
\ln \frac{1}{r_{m}} \leqslant C_{15}\left(\alpha, s^{+}, \beta^{+}\right) \ln m .
$$

Поэтому для $m \geqslant 3$

$$
\left(r_{m}\right)^{s^{+}}\left(\ln \frac{1}{r_{m}}\right)^{n-s^{+}} \geqslant \frac{C_{14}\left(C_{15}\right)^{n-s^{+}}}{m(\ln m)^{s^{+}-n}} \geqslant \frac{C_{14}\left(C_{15}\right)^{n-s^{+}}}{m \ln m}
$$

и теорема доказана. Рассмотрим теперь случай, когда $s^{+}>n+1$. Из $(5)$ для $z \in(0, b)$ имеем

$$
\alpha(z) \geqslant 4 \lambda_{1} \ln \ln \frac{1}{z}-4 \lambda_{1} \ln \ln \frac{1}{b}-\int_{0}^{b} \sigma(t) d t .
$$


Таким образом,

$$
r_{m} \leqslant \exp \left(-\frac{1}{2 M_{1}}\left(4 \lambda_{1} \ln \ln \frac{1}{z}-C_{16}\left(\alpha, \lambda_{1}\right)\right)\right)=C_{17}\left(\alpha, \lambda_{1}, M_{1}\right)\left(\ln \frac{1}{z_{m}}\right)^{-2 \lambda_{1} / M_{1}} .
$$

Учитьвая, что $z_{m} \leqslant e^{-m}$, получаем

$$
\left(r_{m}\right)^{s^{+}} \leqslant C_{18}\left(\alpha, s^{+}, \lambda_{1}, M_{1}\right) m^{-1} .
$$

С другой стороны,

$$
\left(\ln \frac{1}{r_{m}}\right)^{n-s^{+}} \leqslant \frac{C_{19}\left(\alpha, s^{+}, \beta^{+}, n\right)}{(\ln m)^{s^{+}-n}}
$$

т.e.

$$
\left(r_{m}\right)^{s^{+}}\left(\ln \frac{1}{r_{m}}\right)^{n-s^{+}} \leqslant \frac{C_{18} C_{19}}{m(\ln m)^{s^{+}-n}}
$$

и условие (32) не может быть выполнено, так как $s^{+}-n>1$. Аналогично рассматривается случай $J<4 \lambda_{1}$. Пусть теперь $J>4 \lambda_{1}$. Тогда существуют константы $K^{\prime}>K^{\prime \prime}>$ $4 \lambda_{1}$ такие, что для достаточно малых $z$

$$
\left|\alpha^{\prime}(z)\right| \geqslant \frac{K^{\prime}}{z \ln (1 / z)} \quad \text { и } \quad \alpha(z) \geqslant K^{\prime \prime} \ln \ln \frac{1}{z} .
$$

Отсюда получаем

$$
r_{m} \leqslant \exp \left(-\frac{K^{\prime \prime}}{2 M_{1}} \ln \ln \frac{1}{z_{m}}\right)=\left(\ln \frac{1}{z_{m}}\right)^{-K^{\prime \prime} /\left(2 M_{1}\right)} \leqslant m^{-K^{\prime \prime} /\left(2 M_{1}\right)} .
$$

Поэтому

$$
\left(r_{m}\right)^{s^{+}} \leqslant m^{-K^{\prime \prime} /\left(4 \lambda_{1}\right)}
$$

и при $s^{+} \geqslant n$ условие (32) не может быть выполнено. Если же $s^{+}>n$, то в силу неубывания при достаточно малых $z$ функции $z^{s^{+}}(\ln (1 / z))^{n-s^{+}}$имеем

$$
\left(r_{m}\right)^{s^{+}}\left(\ln \frac{1}{r_{m}}\right)^{n-s^{+}} \leqslant\left(\frac{K^{\prime \prime}}{2 M_{1}}\right)^{n-s^{+}} m^{-K^{\prime \prime} /\left(4 \lambda_{1}\right)}(\ln m)^{n-s^{+}} .
$$

Так как $K^{\prime \prime}>4 \lambda_{1}$, то вновь условие (32) не выполняется. Теорема доказана.

Обозначим для натурального $m D \cap\left\{(x, t):-z_{m}<t<0\right\}$ и $\partial D \cap\left\{(x, t):-z_{m}<t<0\right\}$ через $D_{m}$ и $\Gamma_{m}$ соответственно.

Теорема 3. Пусть $u(x, t)$ - положительная L-субпараболическая в $D_{m}$ функиия, непрерывная в $\overline{D_{m}}$ и обращающаяся в нуль на $\Gamma_{m}$. Тогда при выполнении условий (2)-(5) и (31) существуют положительные константы $K_{1}$ и $\gamma$, зависящие лишь от $M_{1}, \lambda_{1} n$ и функиии $\alpha(z)$, такие, что если $m$ достаточно велико $u$ натуральное число $k(m)$ не меньше $K_{1} \ln \ln m$, то

$$
\sup _{D_{m}} u \geqslant\left(1+\eta e^{m s^{+}} p^{+}\left(B_{m}^{+}\right)\right) \sup _{D_{m}+k(m)} u .
$$


ДокАЗАТЕльство. Пусть $H_{m}$ имеет тот же смысл, что и раньше, а $\left(s^{+}, \beta^{+}\right)$-допустимая мера $\nu_{m}$ на $H_{m}$ такова, что

$$
\nu_{m}\left(H_{m}\right) \geqslant \frac{1}{2} p^{+}\left(H_{m}\right)
$$

Обозначим $\sup _{D_{m}}$ через $M_{m}$, и пусть $F_{m}(x, t)=M_{m}\left(1-W_{m}(x, t)\right)$, где

$$
W_{m}(x, t)=\int_{H_{m}} G^{+}(x-y, t-\tau) d \nu_{m}(y, \tau) .
$$

Ясно, что $F_{m} \geqslant 0$ на $\Gamma_{m}$, а на $N_{m}=\partial D_{m} \cap\left\{(x, t): t=-z_{m}\right\}$ функция $W_{m}$ обращается в нуль, т.е. там $F_{m}=M_{m}$. По принципу максимума $u(x, t) \leqslant F_{m}(x, t)$ в $D_{m}$ и, в частности,

$$
M_{m+k(m)} \leqslant \sup _{D_{m+k(m)}} F_{m}=M_{m}\left(1-\inf _{N_{m+k(m)}} W_{m}\right)
$$

Оценим сейчас $\inf _{N_{m+k(m)}} W_{m}$. Пусть $(x, t) \in N_{m+k(m)},(y, \tau) \in H_{m}$ и $m$ настолько велико, что $k(m) \geqslant 3$. Имеем

$$
\begin{aligned}
& \exp \left(-\frac{|x-y|^{2}}{4 \beta^{+}\left(-z_{m+k(m)}-\tau\right)}\right) \\
& \quad \geqslant \exp \left(-\frac{|y|^{2}}{4 \beta^{+}\left(-z_{m+k(m)}-\tau\right)}\right) \cdot\left(\exp \left(-\frac{|y|^{2}}{4 \beta^{+}\left(-z_{m+k(m)}-\tau\right)}\right)\right)^{(|x|+|y|)^{2} /|y|^{2}-1} \\
& \quad \geqslant \exp \left(-\frac{|y|^{2}}{4 \beta^{+}\left(-z_{m+k(m)}-\tau\right)}\right) \cdot\left(\exp \left(-\frac{|y|^{2}}{4 \beta^{+}\left(-z_{m+k(m)}-\tau\right)}\right)\right)^{3|x| /|y|} \\
& \quad=\exp \left(-\frac{|y|^{2}}{4 \beta^{+}\left(-z_{m+k(m)}-\tau\right)}\right) \cdot J_{m}^{1} .
\end{aligned}
$$

С другой стороны, для достаточно больших $m$

$$
\begin{aligned}
3 \frac{|x|}{|y|} & \leqslant 3 \sqrt{\frac{z_{m+k(m)} \ln \left(1 / r_{m+k(m)}\right)}{z_{m+1} \ln \left(1 / r_{m+1}\right)}}=3 b_{m}, \\
\exp \left(-\frac{|y|^{2}}{4 \beta^{+}\left(-z_{m+k(m)}-\tau\right)}\right) & \geqslant\left(\exp \left(-\frac{|y|^{2}}{4 \beta^{+}(-\tau)}\right)\right)^{z_{m} /\left(z_{m}-z_{m+k(m)}\right)} \\
& \geqslant\left(e^{m s^{+}}\left(z_{m+2}\right)^{s^{+}}\right)^{2} \geqslant C_{20}\left(\alpha, s^{+}\right)\left(e^{m s^{+}}\left(z_{m}\right)^{s^{+}}\right)^{2} .
\end{aligned}
$$

Поэтому, учитьвая, что $b_{m} \leqslant C_{21}(\alpha, n)$, получаем

$$
\begin{gathered}
J_{m}^{1} \geqslant C_{22}\left(\alpha, s^{+}, n\right)\left(e^{m s^{+}}\left(z_{m}\right)^{s^{+}}\right)^{6 b_{m}}, \quad \text { т.е. } \\
\ln \frac{C_{22}}{J_{m}^{1}} \leqslant 6 s^{+} b_{m} \ln \frac{1}{r_{m}} \leqslant C_{23}\left(\alpha, s^{+}\right) \sqrt{\frac{z_{m+k(m)}}{z_{m}} \ln \frac{1}{r_{m+k(m)}} \ln \frac{1}{r_{m}}} .
\end{gathered}
$$

Используя (13), имеем

$$
\begin{aligned}
\ln \frac{1}{r_{m+k(m)}} & =\ln \frac{1}{r_{m}}+\ln \left(\exp \left(\frac{1}{2 M_{1}}\left(\alpha\left(z_{m+k(m)}\right)-\alpha\left(z_{m}\right)\right)\right)\right) \\
& \leqslant \ln \frac{1}{r_{m}}+\frac{K}{2 M_{1}} \ln \frac{z_{m}}{z_{m+k(m)}} \leqslant \ln \frac{1}{r_{m}}+\left(\frac{z_{m}}{z_{m+k(m)}}\right)^{K /\left(2 M_{1}\right)} .
\end{aligned}
$$


Так как $a_{1}+a_{2} \leqslant 2 a_{1} a_{2}$ при $a_{1} \geqslant 1, a_{2} \geqslant 1$, то

$$
\ln \frac{1}{r_{m+k(m)}} \leqslant 2\left(\frac{z_{m}}{z_{m+k(m)}}\right)^{K /\left(2 M_{1}\right)} \ln \frac{1}{r_{m}} .
$$

Обозначая $\frac{1}{2}\left(1-K /\left(2 M_{1}\right)\right)$ через $\delta>0$, из последней оценки и $(38)$ получаем

$$
\ln \frac{C_{22}}{J_{m}^{1}} \leqslant 2 C_{23}\left(\frac{z_{m}}{z_{m+k(m)}}\right)^{\delta} \ln \frac{1}{r_{m}} \leqslant 2 C_{23} \exp [-\delta k(m)] \ln \frac{1}{r_{m}} .
$$

Имеем далее

$$
\ln \frac{1}{r_{m}}=\frac{\alpha\left(z_{m}\right)}{2 M_{1}} \leqslant \frac{\alpha\left(e^{-m}\right)}{2 M_{1}}+\frac{K}{2 M_{1}} \ln \frac{1}{r_{m}}
$$

т.e.

$$
\ln \frac{1}{r_{m}} \leqslant \frac{1}{4 M_{1} \delta} \alpha\left(e^{-m}\right) .
$$

Как видно из доказательствапредыдушей теоремы, условие (31) не может вьполняться, если $J>4 \lambda_{1}$. Таким образом, для достаточно малых $z \alpha(z) \leqslant 5 \lambda_{1} \ln \ln (1 / z)$ и поэтому

$$
\alpha\left(e^{-m}\right) \leqslant 5 \lambda_{1} \ln m \text {. }
$$

Положим $K_{1}=1 / \delta$. Учитьвая теперь $(40),(41)$ и условие $k(m) \geqslant K_{1} \ln \ln m$, из (39) получаем

$$
\ln \frac{C_{22}}{J_{m}^{1}} \leqslant \frac{3 C_{23}}{M_{1} \delta}(\ln m)^{1-\delta K_{1}} \leqslant \frac{3 C_{23}}{M_{1} \delta} .
$$

Таким образом,

$$
J_{M}^{1} \geqslant C_{22} \exp \left(-\frac{3 C_{23}}{M_{1} \delta}\right)=C_{24}
$$

что вместе с (37) влечет за собой

$$
W_{m}(x, t) \geqslant C_{24} W_{m}\left(0,-z_{m+k(m)}\right), \quad(x, t) \in N_{m+k(m)} .
$$

Имеем далее для $(y, \tau) \in H_{m}$

$$
\begin{aligned}
& \exp \left(-\frac{|y|^{2}}{4 \beta^{+}\left(-z_{m+k(m)}-\tau\right)}\right) \\
& \quad=\exp \left(-\frac{|y|^{2}}{4 \beta+(-\tau)}\right)\left(\exp \left(-\frac{|y|^{2}}{4 \beta+(-\tau)}\right)\right)^{z_{m+k(m)} /\left(-z_{m+k(m)}-\tau\right)} \\
& \quad=\exp \left(-\frac{|y|^{2}}{4 \beta+(-\tau)}\right) J_{m}^{2}
\end{aligned}
$$

С другой стороны, для достаточно больших $m$

$$
\begin{aligned}
J_{m}^{2} & \geqslant\left(\exp \left(-\frac{|y|^{2}}{4 \beta^{+}(-\tau)}\right)\right)^{2 z_{m+k(m)} / z_{m}} \\
& \geqslant\left(\exp \left(-s^{+} \ln \frac{e^{-m}}{z_{m+2}}\right)\right)^{2 z_{m+k(m)} /\left(2 z_{m}\right)} \geqslant\left(\exp \left(-2 s^{+} \ln \frac{1}{r_{m}}\right)\right)^{2 z_{m+k(m)} /\left(2 z_{m}\right)}
\end{aligned}
$$


т.е. с учетом (40) и (41)

$$
\begin{aligned}
\ln \frac{1}{J_{m}^{2}} & \leqslant 4 s^{+} \frac{z_{m+k(m)}}{z_{m}} \ln \frac{1}{r_{m}} \leqslant \frac{s^{+}}{M_{1} \delta} \exp [-k(m)] \alpha\left(e^{-m}\right) \\
& \leqslant \frac{5 \lambda_{1} s^{+}}{M_{1} \delta}(\ln m)^{1-K_{1}} \leqslant \frac{5 \lambda_{1} s^{+}}{M_{1} \delta}
\end{aligned}
$$

так как $K_{1}=1 / \delta>1$. Таким образом,

$$
J_{m}^{2} \geqslant \exp \left(-\frac{5 \lambda_{1} s^{+}}{M_{1} \delta}\right)=C_{25}
$$

поэтому, используя $(42),(43),(35),(23)$ и $(16)$, заключаем, что

$$
\begin{aligned}
\inf _{N_{m+k(m)}} W_{m}(x, t) & \geqslant C_{24} C_{25} W_{m}(0,0) \geqslant C_{24} C_{25} e^{m s^{+}} \nu_{m}\left(H_{m}\right) \\
& \geqslant \frac{C_{24} C_{25}}{2} e^{m s^{+}} p^{+}\left(H_{m}\right) \geqslant \frac{C_{24} C_{25} C_{6}}{2 C_{1}} e^{m s^{+}} p^{+}\left(B_{m}^{+}\right)
\end{aligned}
$$

Полагая теперь $\eta=C_{24} C_{25} C_{6} /\left(2 C_{1}\right)$, из (36) и (44) получаем требуемую оценку (34). Теорема доказана.

СлЕДСТВИЕ 2. Пусть для натурального $i \geqslant 3 \quad m_{i}=\left[4 K_{1} i \ln \ln i\right]+1$. Тогда если $u(x, t)$ - положительная $L$-субпараболическая в $D_{m_{i}}$ функция, непрерывная в $D_{m_{i}}$ и обращающаяся в нуль на $m_{i}$, то для достаточно больших $і$ при выполнении условий (2)-(5) и (31)

$$
\sup _{D_{m_{i}}} u \geqslant\left(1+\eta e^{m_{i} s^{+}} p^{+}\left(B_{m_{i}}^{+}\right)\right) \sup _{D_{m_{i+1}}} u .
$$

Для доказательства достаточно заметить, что при достаточно больших $i$

$$
m_{i+1}-m_{i} \geqslant K_{1} \ln \ln m_{i}
$$

\section{4. Достаточное условие регулярности.}

Теорема 4. Если относительно коэффициентов оператора $L$ и области $D$ справедливы предположения (2)-(5), то для регулярности точки $(0,0)$ относительно первой краевой задачи достаточно, чтобы выполнялосв условие (31).

ДокАЗАТЕЛьСТво. Вначале покажем, что если

$$
\sum_{m=3}^{\infty} \frac{1}{\ln \ln m} e^{m s^{+}} p^{+}\left(B_{m}^{+}\right)=\infty
$$

то

$$
\sum_{i=3}^{\infty} e^{m_{i} s^{+}} p^{+}\left(B_{m_{i}}^{+}\right)=\infty
$$


Так как для любой непрерьвной и монотонно убьвающей на $[1, \infty)$ положительной функции $\varphi(z)$, стремящейся к нулю при $z \rightarrow \infty$, при всяком натуральном $i>1$

$$
\sum_{k=2}^{i} \varphi(k) \leqslant \int_{1}^{i} \varphi(z) d z \leqslant \sum_{k=1}^{i-1} \varphi(k)
$$

то для достаточно больших натуральных $q$

$$
\sum_{i=3}^{q} e^{m_{i} s^{+}} p^{+}\left(B_{m_{i}}^{+}\right) \geqslant C_{26}\left(\alpha, s^{+}, n\right) \int_{3}^{q+1} e^{m(t) s^{+}} p^{+}\left(B_{\{m(t)\}}^{+}\right) d t .
$$

Здесь $m(t)=4 K_{1} t \ln \ln t, B_{\{z\}}^{+}=\left\{(x, t): e^{z s^{+}} \leqslant G^{+}(-x,-t) \leqslant e^{(z+1) s^{+}}\right\} \backslash D$. Но, с другой стороны,

$$
\begin{aligned}
\int_{3}^{q+1} e^{m(t) s^{+}} p^{+}\left(B_{\{m(t)\}}^{+}\right) d t & \geqslant \frac{1}{8 K_{1}} \int_{12 K_{1} \ln \ln 3}^{4 K_{1}(q+1) \ln \ln (q+1)} e^{z s^{+}} p^{+}\left(B_{\{z\}}^{+}\right) \frac{d z}{\ln \ln z} \\
& \geqslant \frac{1}{8 K_{1}} \sum_{m=\left[12 K_{1} \ln \ln 3\right]+2}^{\left[4 K_{1} q \ln \ln q\right]} \frac{1}{\ln \ln m} e^{m s^{+}} p^{+}\left(B_{m}^{+}\right) .
\end{aligned}
$$

Теперь из (47), (48) видно, что вьполнение условия (45) влечет за собой справедливость (46). Отсюда, используя теорему 2 , заключаем, что при выполнении условия (31) верно (46). Для доказательства регулярности точки $(0,0)$ достаточно показать следующее: для любых $\varepsilon_{1}>0$ и $\varepsilon_{2}>0$ найдется $\delta>0$ такое, что какова бы ни была $L$-субпараболическая в $D$ функция $u(x, t) \leqslant 1$, обращающаяся в нуль на $\partial D \cap\{(x, t)$ : $\left.-\varepsilon_{1}<t<0\right\}$, для $(x, t) \in D \cap\{(x, t):-\delta<t<0\}$ вьполнено неравенство $u(x, t)<\varepsilon_{2}$ (см. [1]). Пусть числа $\varepsilon_{1}, \varepsilon_{2}$ и функция $u(x, t)$ уже заданы. Обозначим через $j \geqslant 3$ наименьшее натуральное число, для которого $z_{m_{j}}<\varepsilon_{1}$, и пусть натуральное число $k>j+1$ таково, что в $D_{m_{k}}$ найдется точка $\left(x^{0}, t^{0}\right)$, в которой

$$
u\left(x^{0}, t^{0}\right) \geqslant \varepsilon_{2} \text {. }
$$

Применяя последовательно следствие из теоремы 3 и используя (49), получаем

$$
1 \geqslant M_{m_{j}} \geqslant \prod_{i=j}^{k-1}\left(1+\eta e^{m-i s^{+}} p^{+}\left(B_{m_{i}}^{+}\right)\right) \cdot \varepsilon_{2}
$$

т.е.

$$
\sum_{i=j}^{k-1} \ln \left(1+\eta e^{m_{i} s^{+}} p^{+}\left(B_{m_{i}}^{+}\right)\right) \leqslant \ln \frac{1}{\varepsilon_{2}} .
$$

Но для всякого натурального $m B_{m}^{+} \subset A_{m}^{+} \subset C_{0, \sqrt{2 M_{1} / e} e^{-m / 2}}^{-e^{-m}, 0}$, поэтому

$$
p^{+}\left(B_{m}\right) \leqslant C_{26}\left(s^{+}, \beta^{+}, n\right) e^{m s^{+}} \text {. }
$$

Пусть

$$
C_{27}=\inf _{t \in\left(0, C_{26}\right]} \frac{\ln (1+\eta t)}{t} .
$$


Из (50) имеем

$$
\sum_{i=j}^{k-1} e^{m_{i} s^{+}} p^{+}\left(B_{m_{i}}^{+}\right) \leqslant \frac{1}{C_{27}} \ln \frac{1}{\varepsilon_{2}} .
$$

В силу условия (46) последнее неравенство может выполняться лишш при $k \leqslant l\left(\varepsilon_{1}, \varepsilon_{2}, \alpha\right.$, $\left.s^{+}, \beta^{+}, n\right)$. Теперь достаточно выбрать $\delta=z_{m_{l}+1}$ и теорема доказана.

СлЕДСТВИЕ 3. Если $L$ - оператор теплопроводности $\Delta-\partial / \partial t$, mo $s^{+}=s^{-}=n / 2$, $\beta^{+}=\beta^{-}=1, B_{m}^{+}=B_{m}^{-}=B_{m}, u$ для регулярности точки $(0,0)$ необходимо $u$ достаточно, чтоби

$$
\sum_{m=1}^{\infty} e^{m n / 2} p\left(B_{m}\right)=\infty .
$$

Последнее условие представляет собой критерий регулярности граничной точки для уравнения теплопроводности, полученньй Эвансом и Гарипи в [14].

\section{СПИСОК ЦИТИРОВАННОЙ ЛИТЕРАТУРЫ}

[1] Ландис Е. М. Уравнения второго порядка эллиптического и параболического типов. М.: Наука, 1971.

[2] Wiener N. The Dirichlet problem // J. Math. Phys. 1924. № 3. P. 127-146.

[3] Petrowsky I. G. Zur ersten Randwertaufgabe de Wärmeleitungsgleichung // Comp. Math. 1935. № 1. P. 383-419.

[4] Ландис Е. М. Необходимое и достаточное условие регулярности граничной точки для задачи Дирихле для уравнения теплопроводности // Докл. АН СССР. 1969. Т. 185. № 3. C. $517-520$.

[5] Ландис Е. М. О регулярности граничной точки для уравнения теплопроводности // Качественная теория краевых задач математической физики. Баку: Элм, 1991. С. 69-96.

[6] Новрузов А.А. О некоторых критериях регулярности граничных точек для линейных и квазилинейных параболических уравнений // Докл. АН СССР. 1973. Т. 209. № 4. С. 785-787.

[7] Ибрагимов А. И. О некоторых качественных свойствах решений уравнений параболического типа второго порядка с непрерьвными коэффициентами // Дифференц. уравнения. 1982. Т. 18. № 2. С. $306-319$.

[8] Мамедов И. Т. О регулярности граничных точек для линейных и квазилинейных уравнений параболического типа // Докл. АН СССР. 1975. Т. 223. №3. С. 559-561.

[9] Мамедов И. Т. О регулярности граничных точек для линейных уравнений параболического типа // Матем. заметки. 1976. Т. 20. № 5. С. 717-723.

[10] Effros E. G., Kazdan J. L. On the Dirichlet problem for the heat equation // Indiana Univ. Math. J. 1971. V. 20. P. 683-694.

[11] Lanconelli E. Sul problema di Dirichlet per l'equazione del calore // Ann. Math. Pura Appl. 1973. V. 97. P. 83-114.

[12] Михайлова Н. А. О поведении на границе решений параболических уравнений 2-го порядка с непрерывными коэффициентами // Матем. заметки. 1985. Т. 38. №6. С. 816-831.

[13] Мамедов И. Т. Оценка модуля непрерывности решения первой краевой задачи для уравнения теплопроводности в граничной точке // Тр. ИММ АН Азерб. 1996. Т. 5(13). С. 30-50.

[14] Evans L. C., Gariepy R. F. Wiener's criterion for the heat equation // Arch. Rath. Mech. Ann. 1992. V. 78. № 4. P. 293-314.

[15] Mamedov I. T. Some notions on parabolic potentials and capacities // Trans. Acad. Sci. Azerb. 1998. V. 18. № 3-4. P. 115-127. 